\title{
Intra-MRI Extraction of Diagnostic Electrocardiograms Using Carotidal Magnetohydrodynamic Voltages
}

\author{
T. Stan Gregory ${ }^{1}$, Kevin James $\mathrm{Wu}^{1}{ }^{1}$, John N. Oshinski ${ }^{2}$ and Zion Tsz Ho Tse ${ }^{1, *(1)}$ \\ 1 College of Engineering, The University of Georgia, Athens, GA 30602, USA; tsgregory@uga.edu (T.S.G.); \\ kwu@uga.edu (K.J.W.) \\ 2 Radiology, Emory University Hospital, Atlanta, GA 30322, USA; jnoshin@emory.edu \\ * Correspondence: ziontse@uga.edu; Tel.: +1-706-542-4189
}

Received: 26 February 2018; Accepted: 2 May 2018; Published: 6 May 2018

\begin{abstract}
The electrocardiogram (ECG) is commonly utilized for patient monitoring during magnetic resonance imaging (MRI) despite known magnetohydrodynamic voltage (VMHD) overlays, which often eclipse the true sinus rhythm and render the signal to be non-diagnostic. This can complicate MRI gating and at-risk patient monitoring, causing alternative low-fidelity signals to become preferred. We aimed to develop a method of isolating the true sinus rhythm from VMHD in order to enable the use of high-fidelity ECGs during MRI procedures. Twelve-lead ECGs were acquired in two healthy volunteers $(n=2)$ in a 3T MRI scanner, while a secondary single lead monitor was positioned across the left common carotid artery to directly record VMHD while cancelling out the true sinus rhythm. Carotid MHD was used to adaptively train a least mean squares filter to update a 12-lead ECG VMHD template and produce: (1) clean 12-lead ECGs and (2) an accurate stroke volume (SV) estimate. The adaptive filtering method was shown to reduce VMHD in 12-lead ECGs. This was demonstrated by an average cross-correlation of 0.81 across all ECG leads calculated between filtered ECG taken inside the MRI scanner and the ECG taken outside the MRI scanner. Residual noise formed $<5 \%$ of the R-wave amplitude. Additionally, the method required only a short training phase. A method to extract real sinus rhythm beats from intra-MRI 12-lead ECGs was presented and shown to provide accurate dynamic measurements of induced VMHD using flow in the carotid artery as a source of dynamic feedback.
\end{abstract}

Keywords: electrocardiogram; magnetohydrodynamic; physiological monitoring; MRI

\section{Introduction}

The electrocardiogram (ECG), while considered to be a standard in the monitoring of patient heart activity, is known to be of non-diagnostic quality in the isocenter of a magnetic resonance imaging (MRI) scanner [1]. This causes a great level of difficulty in the synchronization of cardiac activity with the MRI scanner during cardiac cine acquisition using QRS complex detection techniques, and in patient monitoring during MRI procedures and guided interventions, especially in the case of high-risk patients such as those with histories of ischemia or stroke [2-5].

Conventional ECG monitoring systems and advanced signal processing techniques have been developed to acquire ECG traces in the presence of gradient artifacts that may occur during MRI scanning procedures $[5,6]$. Despite the prevention of voltages related to MRI gradient ramp signal induction, further ECG distortion is known to occur due to a magnetohydrodynamic (MHD) effect generated by interactions between the static magnetic field of the MRI $\left(\mathrm{B}_{0}\right)$ and blood plasma electrolytes ejected into the aortic arch during early systole [1,3,5,7-11]. 
In an effort to improve the success of cardiac cine acquisition, techniques for QRS complex detection have been developed and validated with MHD voltages (VMHD) superimposed upon ECG traces, such as the 3DQRS method and techniques based in the vectorcardiography (VCG) frame of reference [2], whereas the 12-lead ECG is represented as a dipole moment in the heart [12].

These methods allow for more effective cardiac gating, but they do not allow for separation of the true ECG $\left(\mathrm{ECG}_{\text {real }}\right)$ from VMHD. Several studies have been performed using independent component analysis (ICA), which has demonstrated some success despite being limited through the relatively small number of discrete noise sources considered in the model and the use of simulated VMHD [13-15]. Preliminary studies have been performed to quantify all contributions to the net recorded intra-MRI signal through dictionary learning for sparse approximations (Figure 1a) [16,17]. However, results from this approach have presented difficulties in filter training for ECG $_{\text {real }}$ extraction. The conventional method of $E_{C G}$ real extraction involves the subtraction of ECGs recorded inside the MRI from those recorded outside the MRI (Figure 1b) [18]. This method is flawed because it is based on the assumption that $\mathrm{ECG}_{\text {real }}$ does not vary once the subject is placed inside the MRI, which is not necessarily the case.

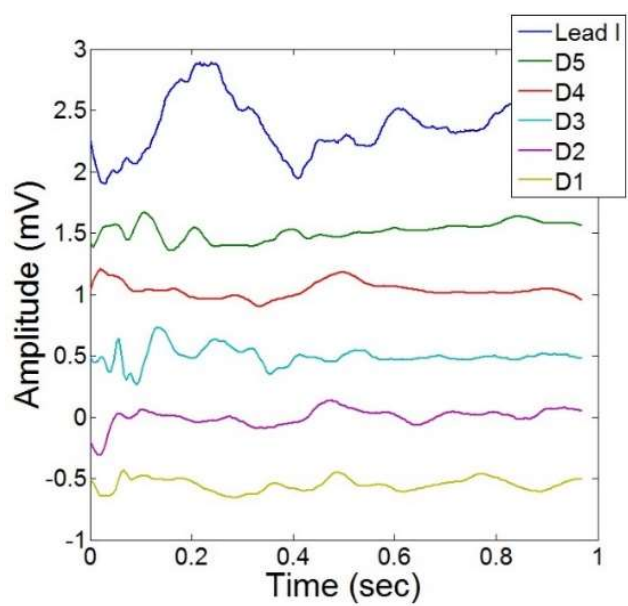

(a)

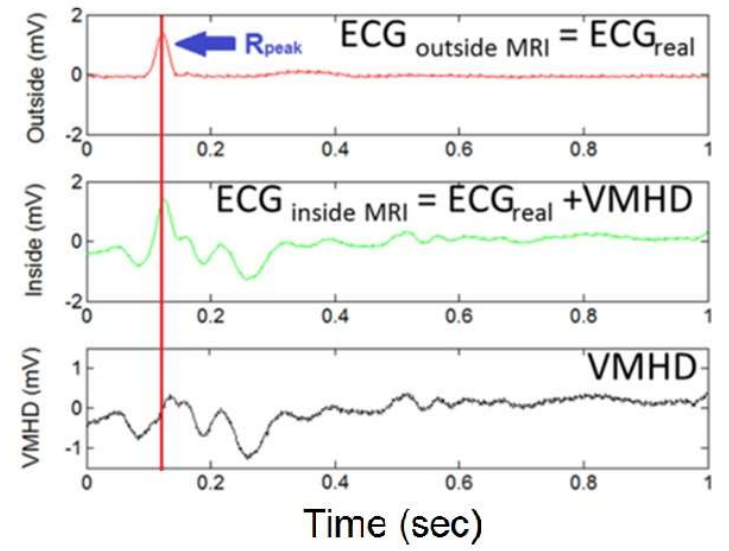

(b)

Figure 1. Conventional methods of $E C_{\text {real }}$ extraction during intra-MRI ECG acquisition. (a) Sparse-encoded dictionary learning method for separation of contributions to the net magnetohydrodynamic (MHD) effect (blue line) by dictionary elements (D1-D5) in a single ECG lead (lead I): contributions are attributed to flow along the aortic arch and in peripheral vasculature. (b) Performance of MHD subtraction technique using a constant ECG real template for MHD-ECG separation during 12-lead ECG data acquisition inside the MRI bore, which does not account for variations in $\mathrm{ECG}_{\text {real }}$.

This study proposes a new methodology for extraction of $\mathrm{ECG}_{\text {real }}$ from recordings obtained inside the MRI, improving upon a method of adaptive filtering using a least means square (LMS) implementation. The proposed method utilizes a dynamic source of pure MHD to train adaptive

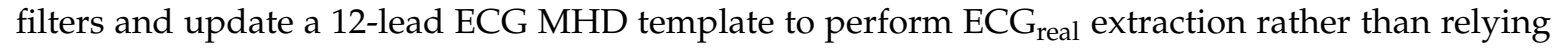
entirely on initial training coefficients without additional feedback. In addition, the training sequence only requires the acquisition of one dataset outside of the MRI bore (head-in), as opposed to prior studies which require two datasets (feet-in and head-in) [5,10], potentially halving the time required for training. This will allow for an increased level of accuracy in ECG $G_{\text {real }}$ extraction and estimation of MHD-derived metrics in real time [19]. 


\section{Materials and Methods}

\subsection{Study Population}

The study was conducted using data acquired from two healthy volunteers during exercise stress testing $(n=2)$ in a Siemens Skyra 3T MRI (Siemens AG, Berlin, Germany). Informed consent was obtained from each subject as per Institutional Review Board standards.

\subsection{Data Acquisition}

Data was recorded to establish a baseline with each subject supine on the scanner table outside of the MRI bore. Each subject was then inserted head-first into the MRI bore until the heart was at the isocenter of the scanner. Twelve-lead ECG traces were acquired during 20-second breath-holds using an ECG recorder modified to be MRI-compatible [5], and a secondary monitor was used to acquire single lead bipolar VMHD signals with approximately 4-inch lead spacing from the subject in the left common carotid artery. Phase contrast MRI (PCMR) cines were similarly acquired in each subject during exercise stress testing in the ascending aorta and in the common carotid artery at rest and during exercise stress to evaluate the relationship between induced VMHD and blood flow in both the ascending aorta and the left common carotid artery. All PCMR cines were obtained with the following parameters: VENC: $150 \mathrm{~cm} / \mathrm{s}$; TR/TE/flip: $37.00 \mathrm{~ms} / 4.00 \mathrm{~ms} / 15^{\circ}$; field-of-view: $300 \mathrm{~mm}$ by $243 \mathrm{~mm}$; slick thickness: $3 \mathrm{~mm}$.

\subsubsection{Correlation between Aortic and Carotidal MHD}

In order to select the proper source of dynamic feedback for the system, the correlation was quantified between induced aortic VMHD extracted from 12-lead ECGs and four MHD sources using a single bipolar lead at the surface of the human body: (1) left common carotid artery (Left-Right and Superior-Inferior lead placement); (2) left femoral artery; (3) across the chest; and the (4) axillary artery. All ECG recordings were acquired inside a 3T MRI. Correlation between extracted MHD from 12-lead ECGs recorded inside the MRI and each source was computed and used to determine the optimal source of intra-MRI dynamic feedback. Furthermore, average flow was quantified in the ascending aorta and the carotid artery using conventional PCMR during exercise stress testing to predict induced VMHD based on superimposing an MHD term on 12-lead ECG traces acquired outside of the MRI bore, whereas MRI derived flow $(q)$ and a unique proportionality constant $\left(\alpha_{i}\right)$ for each lead $(i=1: 12)$ were used to predict induced VMHD which occurs in 12-lead ECG (Equation (1)) [18].

$$
V M H D=\alpha_{i} B_{0} q \text {, for leads } i=1: 12 \text {. }
$$

Predicted VMHD values based on MRI-derived blood flow and actual VMHD measured from body surface electrodes were analyzed statistically to justify usage of carotidal MHD as the dynamic feedback term.

\subsubsection{Adaptive Filter Training}

In order to properly utilize the dynamic feedback provided from the carotidal MHD, twelve adaptive LMS filters were trained (length, step size) to update the morphology of the carotidal MHD to match the morphology of VMHD obtained from 12-lead ECGs (aortic MHD). An aortic MHD template was obtained from the subtraction of 12-lead ECGs obtained inside and outside of the MRI bore. The aortic MHD template obtained at the baseline heart rate was used to update the morphology of carotidal MHD (Figure 2a (top)). Carotidal MHD was phase compensated to match VMHD obtained from the 12-lead ECG (Figure 2b), and input into an LMS adaptive filter to train the LMS adaptive filter coefficients to minimize the least mean squares error between the two signals. The dynamic feedback generated from the carotidal MHD allows for the updated 12-lead aortic MHD to respond more quickly to changes in cardiac activity and reduce error associated with variations in heartbeats. 
Following the initial training of the LMS filter coefficients, the aortic MHD template can be updated

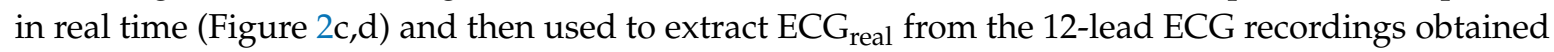
in real time inside the MRI bore, and to obtain a more accurate estimate of MHD-derived metrics as previously demonstrated for stroke volume (SV) estimation (Figure 2a (bottom)) [11,19].

To evaluate the proposed method, a twelve-lead ECG was acquired inside the MRI bore and ECG $_{\text {real }}$ extraction was performed. Then a twelve-lead ECG was acquired outside the MRI bore to set the "gold standard." The correlation was determined between the filtered ECG obtained inside the MRI bore and the unfiltered ECG obtained outside the MRI bore.

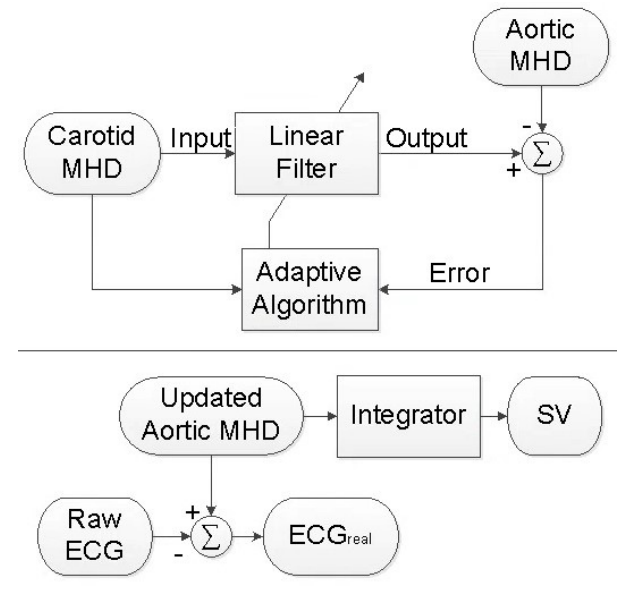

(a)

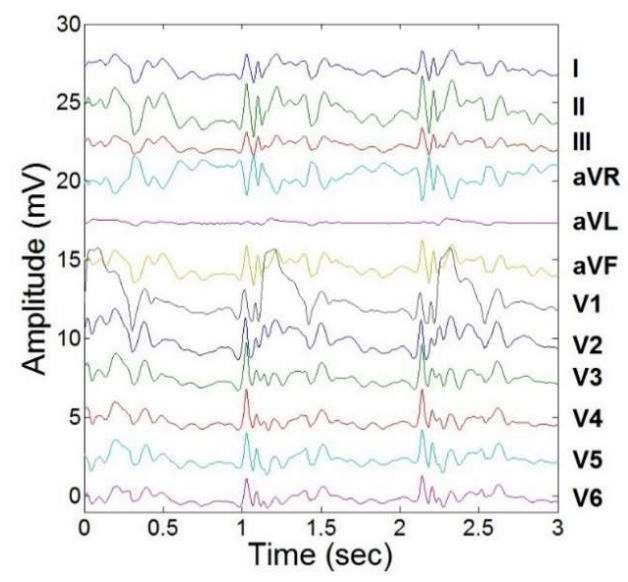

(c)

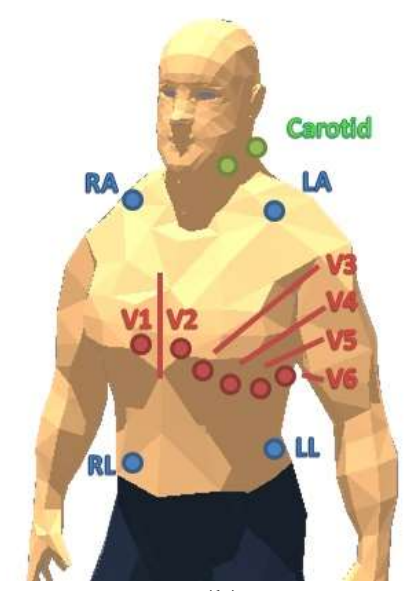

(b)

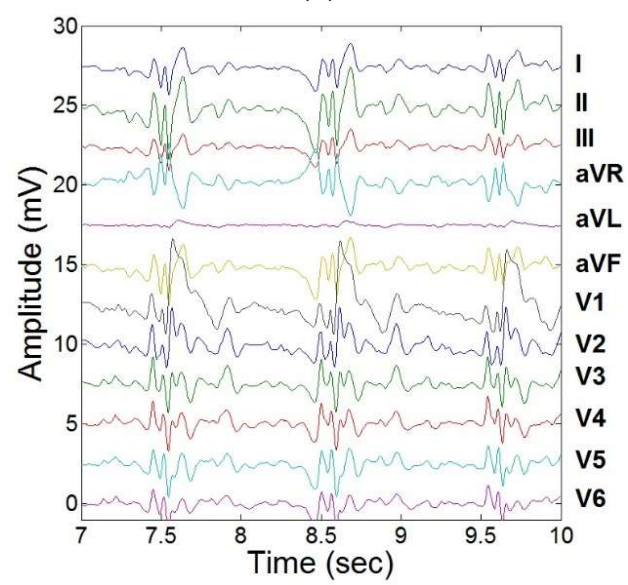

(d)

Figure 2. Development of a dynamic feedback method for higher accuracy real-time 12-lead ECG monitoring and estimation of MHD-derived SV inside the MRI bore. (a) (Top) Adaptive filtering method to morph the carotid artery MHD trace into the aortic MHD trace. (Bottom) $\mathrm{ECG}_{\text {real }}$ and SV estimation derived from aortic MHD developed from carotidal MHD. (b) Single bipolar carotidal lead and 12-lead ECG placement. (c) 12-lead ECG acquired inside the 3T MRI. Each lead contains ECG real and induced MHD voltage (VMHD) components. (d) Updating the morphology of carotidal MHD to match aortic MHD using trained adaptive least means square (LMS) filters as acquired in 12-lead ECGs at $3 \mathrm{~T}$.

\subsubsection{Evaluation during Exercise Stress Testing}

In order to evaluate the efficacy of the proposed methodology of dynamic physiological feedback, a series of exercise stress tests were performed in each subject. Following the training stage, subjects were positioned head-first into the bore of the MRI, and baseline scans of the aortic arch and the carotid artery were performed. Three datasets were acquired at varying levels of cardiac activity: (1) baseline; (2) elevated; and (3) return to baseline. An MRI-compatible exercise band was used to elevate each 
subject's heart rate by $50 \%$, at which point data collection was performed. A period of $30 \mathrm{~min}$ after the induced stress was allowed for the subject to return to baseline heart rate. Extraction of ECG $\mathrm{G}_{\text {real }}$ was performed on the dataset during the period of exercise stress testing, and a Pearson's ranked correlation coefficient was calculated to determine efficacy of $\mathrm{ECG}_{\text {real }}$ extraction.

\section{Results}

\subsection{Correlation between Aortic and Carotidal MHD}

Figure 3a shows the VMHD obtained from single lead MHD sources acquired from carotidal (Left-Right and Superior-Inferior lead placements), femoral, chest, and axillary arterial vasculature. The correlations between the VMHD from each single lead MHD source and the VMHD from the 12-lead ECG are plotted in Figure 3b. The conventional PCMR scans of the ascending aorta and the common carotid artery obtained during exercise stress testing are displayed in Figure 3c.

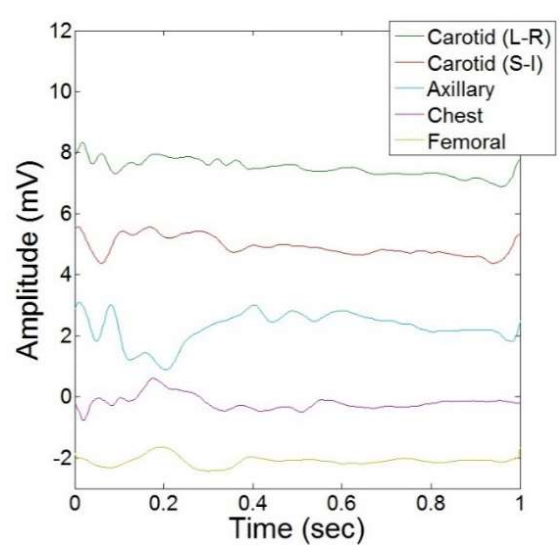

(a)

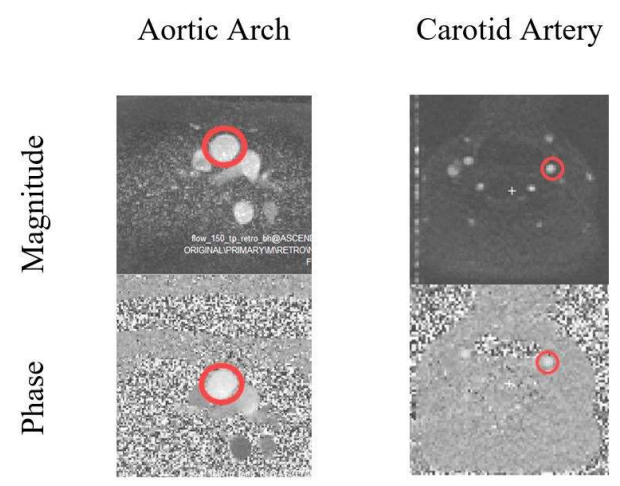

(c)

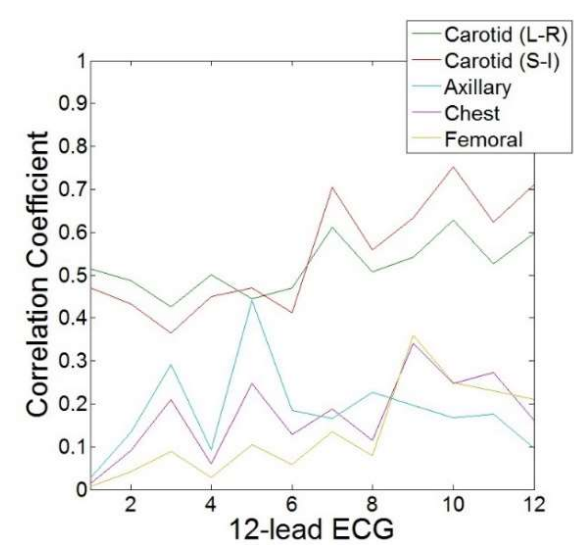

(b)

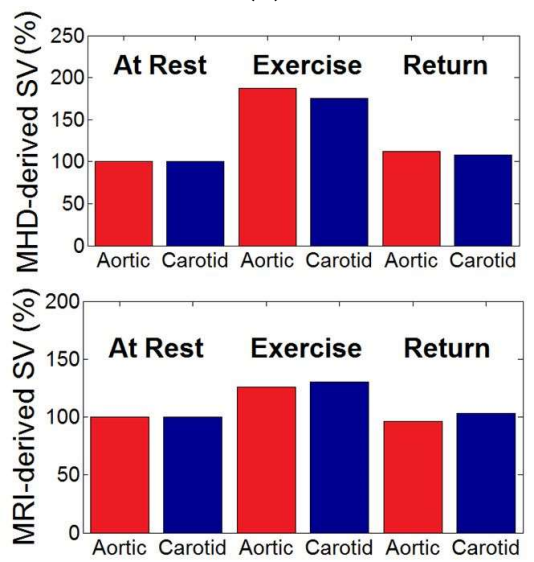

(d)

Figure 3. Demonstration of correlation between induced MHD in the aortic arch and the common carotid artery in the selection of a source of dynamic system feedback. (a) Induced MHD voltages obtained from peripheral vasculature. (b) Cross-correlation index obtained between each peripheral vasculature MHD source and MHD extracted from each lead in the 12-lead ECG. On the $x$-axis, the numbers 1-12 represent the 12 leads used in standard 12-lead ECG in the following order: I, II, III, aVR, aVL, aVF, V1, V2, V3, V4, V5, and V6. This lead numbering system is defined in Table 1. (c) Phase contrast MR (PCMR) transverse plane images of the aortic arch and left common carotid artery used to estimate systolic magnitude (top) and phase (bottom). (d) MHD (top) and flow (bottom) in the aortic arch and the common carotid artery during exercise stress testing, illustrating close relationship between flow in the vessels. 
Extracted flow and VMHD from the carotid artery and the aortic arch were each shown to have a similar increase during exercise stress testing (Figure 3d). MRI-derived flow showed a lower percent increase in SV as compared to MHD-derived flow, which was attributed to the increased acquisition time required by PCMR scanning as opposed to the real-time beat-to-beat estimation provided using VMHD.

Cross-correlation coefficients between MHD obtained from the left common carotid artery and the 12-lead ECG maintained a mean coefficient greater than each alternate peripheral vasculature source, with a minimized standard deviation in correlation coefficients in the Left-Right lead placement as opposed to the Superior-Inferior lead placement (Table 1). The first column in Table 1 defines the numbering system used in this paper for the 12 leads in standard 12-lead ECG.

Table 1. Correlation coefficients obtained between 12-lead MHD and peripheral vasculature.

\begin{tabular}{ccccccc}
\hline Lead Number & Lead Name & Carotid (L-R) & Carotid (S-I) & Axillary & Chest & Femoral \\
\hline 1 & I & 0.51 & 0.47 & 0.03 & 0.01 & 0.01 \\
2 & II & 0.49 & 0.43 & 0.13 & 0.09 & 0.04 \\
3 & III & 0.43 & 0.37 & 0.29 & 0.21 & 0.09 \\
4 & aVR & 0.50 & 0.45 & 0.09 & 0.06 & 0.03 \\
5 & aVL & 0.45 & 0.47 & 0.44 & 0.25 & 0.10 \\
6 & aVF & 0.47 & 0.41 & 0.18 & 0.13 & 0.06 \\
7 & V1 & 0.61 & 0.70 & 0.17 & 0.19 & 0.14 \\
8 & V2 & 0.51 & 0.56 & 0.23 & 0.11 & 0.08 \\
9 & V3 & 0.54 & 0.63 & 0.20 & 0.34 & 0.36 \\
10 & V4 & 0.63 & 0.75 & 0.17 & 0.25 & 0.25 \\
11 & V5 & 0.53 & 0.62 & 0.18 & 0.27 & 0.23 \\
12 & V6 & 0.60 & 0.71 & 0.10 & 0.16 & 0.21 \\
\hline & Mean & 0.52 & 0.55 & 0.18 & 0.17 & 0.13 \\
& Standard & 0.06 & 0.13 & 0.11 & 0.10 & 0.11 \\
\hline
\end{tabular}

\subsection{Adaptive Filter Training}

Figure 4 presents the ECGs acquired to evaluate the $\mathrm{ECG}_{\text {real }}$ extraction method. The unfiltered ECG acquired outside the MRI bore is shown in Figure 4a and the unfiltered ECG acquired inside the MRI bore is shown in Figure $4 \mathrm{~b}$. ECG real $_{\text {was }}$ then extracted from the ECG acquired inside the MRI bore with the filtering technique, and the result is shown in Figure 4c.

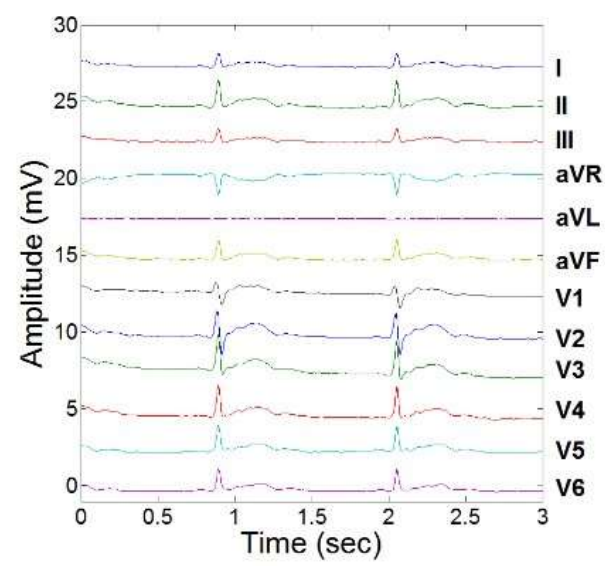

(a)

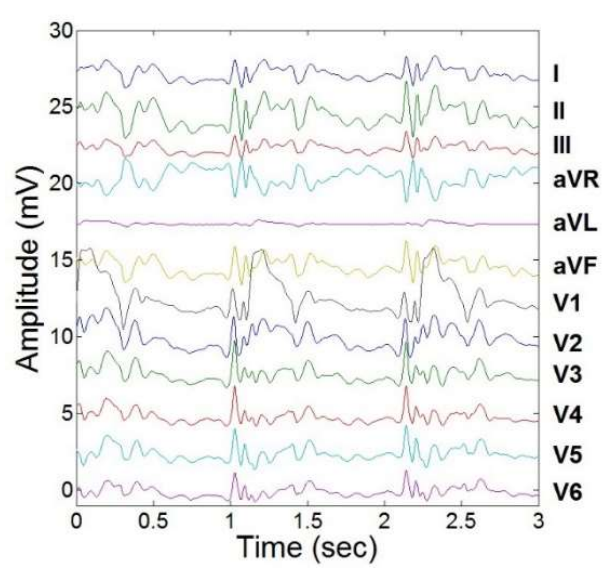

(b)

Figure 4. Cont. 


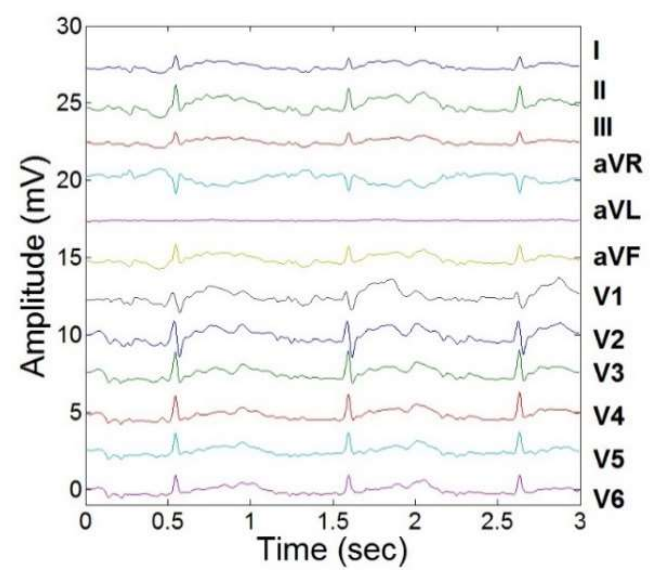

(c)

Figure 4. Training of LMS adaptive filters to remove induced MHD signals, and response to real-time feedback from carotidal MHD. (a) 12-lead ECG acquired outside the MRI bore. (b) 12-lead ECG acquired

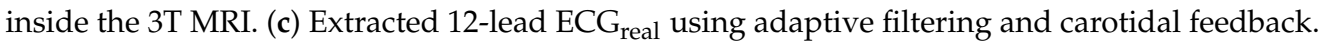

The extracted 12-lead $\mathrm{ECG}_{\text {real }}$ after the filter training stage was found to correlate well with the "gold standard" ECG obtained outside the MRI, with an average cross-correlation index across all leads of 0.81 (Table 2).

Table 2. Cross-correlation coefficients between $\mathrm{ECG}_{\text {real }}$ and $\mathrm{ECG}_{\text {real }}$ extracted intra-MRI.

\begin{tabular}{ccccccccccccc}
\hline Lead & I & II & III & aVR & aVL & aVF & V1 & V2 & V3 & V4 & V5 & V6 \\
\hline $\begin{array}{l}\text { Correlation } \\
\text { Coefficient }\end{array}$ & 0.79 & 0.77 & 0.72 & 0.78 & 0.78 & 0.76 & 0.87 & 0.91 & 0.89 & 0.86 & 0.85 & 0.76 \\
\hline
\end{tabular}

\subsection{Evaluation during Exercise Stress Testing}

The results of $\mathrm{ECG}_{\text {real }}$ extraction performed using carotidal dynamic feedback during exercise stress testing are illustrated in Figure 5. Variations in ECG segments were observed due to the elevated heart rate. This more accurate separation of $\mathrm{ECG}_{\text {real }}$ will in turn allow for increased accuracy in estimating MHD-derived metrics, such as stroke volume. There was less than a $5 \%$ variation in R-peak between filtered ECGs obtained inside the MRI and ECGs obtained outside the MRI scanner in all subjects.

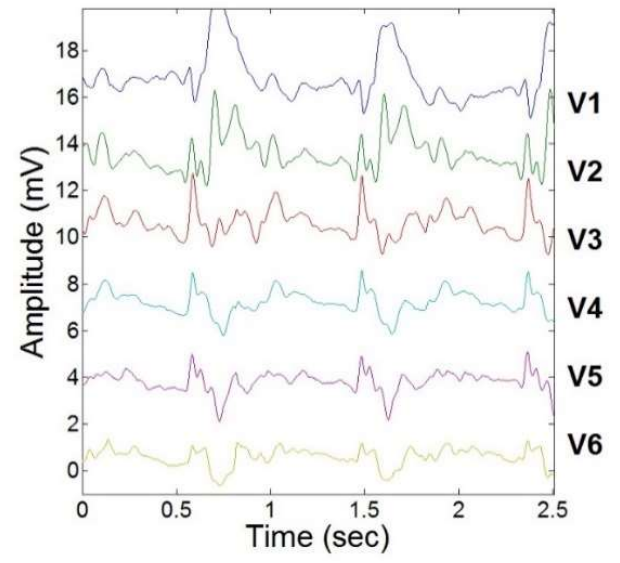

(a)

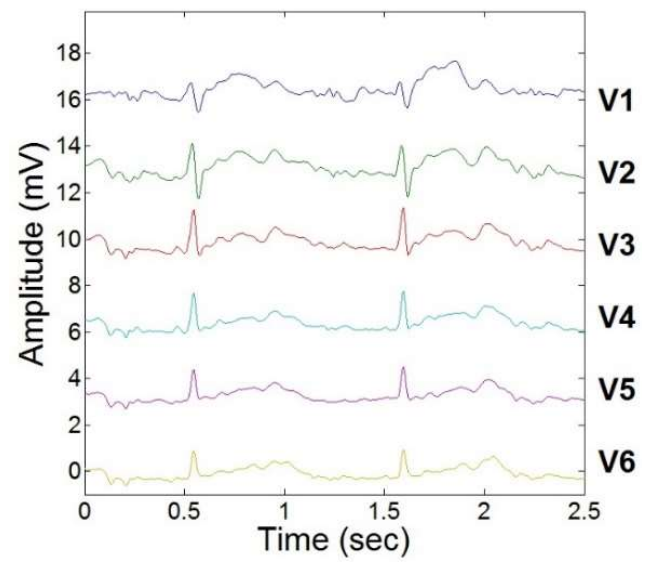

(b)

Figure 5. Cont. 


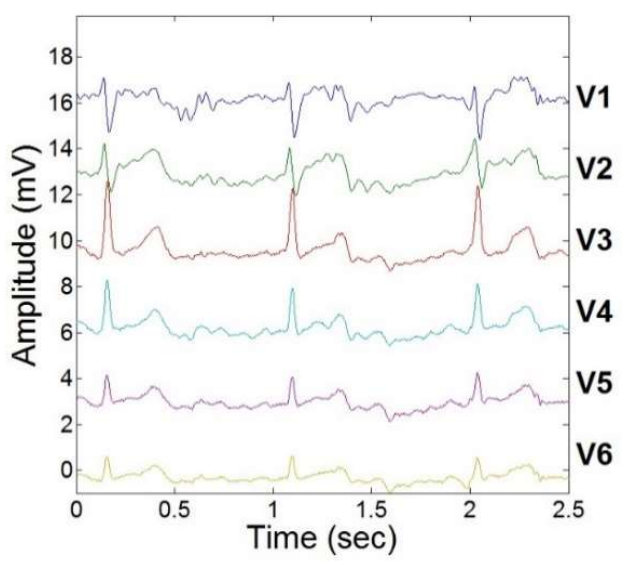

(c)

Figure 5. ECG $\mathrm{E}_{\text {real }}$ extraction during exercise stress testing using adaptive filtering schema and dynamic carotidal VMHD feedback. (a) 12-lead ECG acquired inside the MRI prior to ECG real $_{\text {extraction. (b) }}$ 12-lead ECG acquired inside the MRI during a resting heart rate. (c) 12-lead ECG acquired inside the MRI during exercise.

\section{Discussion}

\subsection{Discussion}

A method to extract ECG signals with reduced VMHD from intra-MRI 12-lead ECGs was presented and shown to provide accurate dynamic measurements of induced VMHD using flow in the carotid artery as a source of dynamic feedback. The addition of the dynamic feedback allows for a shorter filter response time to heartbeat variability, and the potential to more accurately estimate ECG $_{\text {real }}$ and VMHD-derived metrics such as stroke volume.

VMHD was acquired using a single-lead ECG monitor from several different vasculature sources on the human body in order to determine an optimal flow source which induces VMHD with a similar morphology to that observed in 12-lead ECGs. The left common carotid artery was observed to have the highest correlation with regards to signal morphology of the aortic MHD extracted from 12-lead ECGs and was therefore selected as the source of dynamic feedback for training of adaptive filters to more accurately separate ECG $_{\text {real }}$ and VMHD intra-MRI. The adaptive filtering schema presented was able to morph carotidal VMHD to match the aortic MHD template, and provide dynamic cardiac feedback to increase the accuracy of physiological monitoring during exercise stress testing.

This method will allow for advanced physiological monitoring within the MRI and has the potential to reduce the time associated with cardiac imaging by increasing the accuracy of cardiac ECG gating through improved MHD suppression.

\subsection{Limitations}

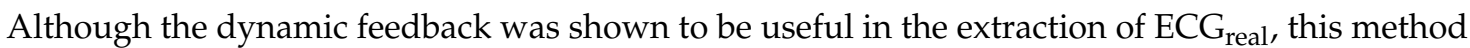
does require the addition of a secondary physiological monitor to provide diagnostic quality ECG traces. This hardware constraint can potentially inhibit the ability of the proposed methodology to become widely used in practice and in conjunction with modern MRI-compatible 12-lead ECG recorders.

\subsection{Future Work}

To more thoroughly and rigorously test the proposed methodology, the experiments should be conducted on a larger group of subjects and expanded to a wider subject population, including individuals of varying physical fitness and gender. Additionally, the subject population should include not only healthy subjects but also subjects with pathologies such as cardiac disorders and arrhythmias. 
This data should then be further evaluated by a cardiologist to validate the ability to acquire diagnostic quality ECGs using the proposed methodology.

\section{Conclusions}

Real-time 12-lead ECGs were acquired and filtered of the magnetohydrodynamic effect through the addition of a dynamic feedback term to a conventional adaptive filtering schema. The feedback term allowed for dynamic changes in induced VMHD to be assessed using a single bipolar lead positioned on the left common carotid artery and for a demonstrated level of accuracy during induced stress.

Author Contributions: Zion Tsz Ho Tse conceived and designed the experiments; T. Stan Gregory and Kevin James $\mathrm{Wu}$ performed the experiments and analyzed the data; John N. Oshinski contributed MRI scanning resources and analysis tools; T. Stan Gregory wrote the paper.

Funding: This research was funded in part by the National Institutes of Health (NIH) Bench-to-Bedside Award, the NIH Center for Interventional Oncology Grant, the National Science Foundation (NSF) I-Corps Team Grant (1617340), NSF REU site program 1359095, the AU-UGA inter-institutional Seed Funding Program, the American Society for Quality Richard J. Schlesinger Grant, the PHS Grant UL1TR000454 from the Clinical and Translational Science Awards Program of the NIH National Center for Advancing Translational Sciences, and the University of Georgia Clinical and Translational Research Unit Seed Grant. This research was also supported by the National Center for Advancing Translational Sciences of the National Institutes of Health under Award Number UL1TR002378. The content is solely the responsibility of the authors and does not necessarily represent the official views of the National Institutes of Health.

Conflicts of Interest: The authors declare no conflict of interest. The founding sponsors had no role in the design of the study; in the collection, analyses, or interpretation of data; in the writing of the manuscript, and in the decision to publish the results.

\section{References}

1. Birkholz, T.; Schmid, M.; Nimsky, C.; Schuttler, J.; Schmitz, B. ECG artifacts during intraoperative high-field MRI scanning. J. Neurosurg. Anesthesiol. 2004, 16, 271-276. [CrossRef] [PubMed]

2. Fischer, S.E.; Wickline, S.A.; Lorenz, C.H. Novel real-time R-wave detection algorithm based on the vectorcardiogram for accurate gated magnetic resonance acquisitions. Magn. Reson. Med. 1999, 42, 361-370. [CrossRef]

3. Gregory, T.S.; Schmidt, E.J.; Zhang, S.H.; Tse, Z.T.H. 3DQRS: A method to obtain reliable QRS complex detection within high field MRI using 12-lead electrocardiogram traces. Magn. Reson. Med. 2014, 71, 1374-1380. [CrossRef] [PubMed]

4. Larson, A.C.; White, R.D.; Laub, G.; McVeigh, E.R.; Li, D.; Simonetti, O.P. Self-gated cardiac cine MRI. Magn. Reson. Med. 2004, 51, 93-102. [CrossRef] [PubMed]

5. Tse, Z.; Dumoulin, C.L.; Clifford, G.D.; Schweitzer, J.; Qin, L.; Oster, J.; Jerosch-Herold, M.; Kwong, R.Y.; Michaud, G.; Stevenson, W.G.; et al. A 1.5T MRI-conditional 12-lead electrocardiogram for MRI and intra-MR intervention. Magn. Reson. Med. 2013, 71, 1336-1347. [CrossRef] [PubMed]

6. Zhang, S.H.; Tse, Z.T.H.; Dumoulin, C.L.; Kwong, R.Y.; Stevenson, W.G.; Watkins, R.; Ward, J.; Wang, W.; Schmidt, E.J. Gradient-induced voltages on 12-lead ECGs during high duty-cycle MRI sequences and a method for their removal considering linear and concomitant gradient terms. Magn. Reson. Med. 2015, 75, 2204-2216. [CrossRef] [PubMed]

7. Blandford, R.; Thorne, K. Magnetohydrodynamics. In Applications of Classical Physics; CalTech: Pasadena, CA, USA, 2004.

8. Gregory, T.S.; Schmidt, E.J.; Zhang, S.H.; Kwong, R.Y.; Stevenson, W.G.; Murrow, J.R.; Tse, Z.T.H. Left-Ventricular Mechanical Activation and Aortic-Arch Orientation Recovered from Magneto-Hydrodynamic Voltages Observed in 12-Lead ECGs Obtained Inside MRIs: A Feasibility Study. Ann. Biomed. Eng. 2014, 42, 2480-2489. [CrossRef] [PubMed]

9. Gupta, A.; Weeks, A.R.; Richie, S.M. Simulation of elevated T-waves of an ECG inside a static magnetic field (MRI). IEEE Trans. Biomed. Eng. 2008, 55, 1890-1896. [CrossRef] [PubMed]

10. Tse, Z.T.H.; Dumoulin, C.L.; Clifford, G.; Jerosch-Herold, M.; Kacher, D.; Kwong, R.; Stevenson, W.G.; Schmidt, E.J. Real-ECG extraction and stroke volume from MR-Compatible 12-lead ECGs; testing during stress, in PVC and in AF patients. J. Cardiovasc. Magn. Reson. 2011, 13, P6. [CrossRef] 
11. Gregory, T.S.; Oshinski, J.; Schmidt, E.J.; Kwong, R.Y.; Stevenson, W.G.; Tse, Z.T.H. Continuous Rapid Quantification of Stroke Volume Using Magnetohydrodynamic Voltages in 3T Magnetic Resonance Imaging. Circ. Cardiovasc. Imaging 2015, 8, e003282. [PubMed]

12. Grishman, A.; Scherlis, L.; Lasser, R.P. Spatial vectorcardiography. Am. J. Med. 1953, 14, 184-200. [CrossRef]

13. Krug, J.; Rose, G.; Clifford, G.; Oster, J. Improved ECG based gating in ultra high field cardiac MRI using an independent component analysis approach. J. Cardiovasc. Magn. Reson. 2013, 15, W33. [CrossRef]

14. Krug, J.; Rose, G.; Stucht, D.; Clifford, G.; Oster, J. Filtering the Magnetohydrodynamic Effect from 12-lead ECG Signals using Independent Component Analysis. Comput. Cardiol. 2012, 589-592.

15. Oster, J.; Pietquin, O.; Abacherli, R.; Kraemer, M.; Felblinger, J. Independent component analysis-based artefact reduction: Application to the electrocardiogram for improved magnetic resonance imaging triggering. Physiol. Meas. 2009, 30, 1381-1397. [CrossRef] [PubMed]

16. Mairal, J.; Bach, F.; Ponce, J.; Sapiro, G. Online learning for matrix factorization and sparse coding. J. Mach. Learn. Res. 2010, 11, 19-60.

17. Yaghoobi, M.; Blumensath, T.; Davies, M.E. Dictionary learning for sparse approximations with the majorization method. IEEE Trans. Signal Process. 2009, 57, 2178-2191. [CrossRef]

18. Oster, J.; Llinares, R.; Payne, S.; Tse, Z.T.H.; Schmidt, E.J.; Clifford, G.D. Comparison of three artificial models of the magnetohydrodynamic effect on the electrocardiogram. Comput. Methods Biomech. Biomed. Eng. 2015, 18, 1400-1417. [CrossRef] [PubMed]

19. Gregory, T.S.; Schmidt, E.J.; Zhang, S.H.; Kwong, R.Y.; Stevenson, W.G.; Oshinski, J.; Tsz Ho Tse, Z. Rapid Quantification of Stroke Volume using Magnetohydrodynamic Voltages in 3T MRI: A Feasibility Study. J. Cardiovasc. Magn. Reson. 2015, 17, P32. [CrossRef]

(C) 2018 by the authors. Licensee MDPI, Basel, Switzerland. This article is an open access article distributed under the terms and conditions of the Creative Commons Attribution (CC BY) license (http:/ / creativecommons.org/licenses/by/4.0/). 